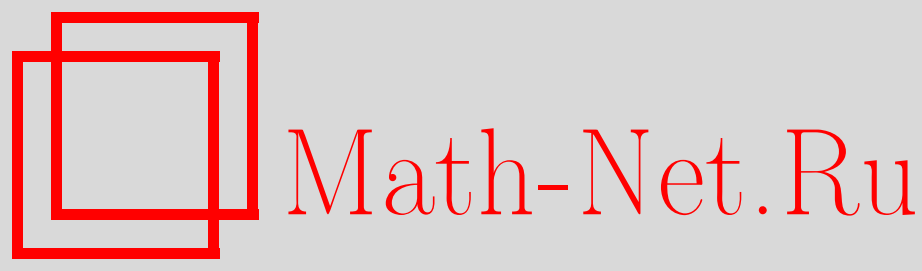

Ж. Аро, Вычисление перенормированной двухточечной корреляционной функции методом адиабатической регуляризации, ТМФ, 2010, том 165, номер 2, 295-307

DOI: https://doi.org/10.4213/tmf6577

Использование Общероссийского математического портала Math-Net.Ru подразумевает, что вы прочитали и согласны с пользовательским соглашением http://www.mathnet.ru/rus/agreement

Параметры загрузки:

IP: 18.207 .199 .55

26 апреля 2023 г., 16:45:20

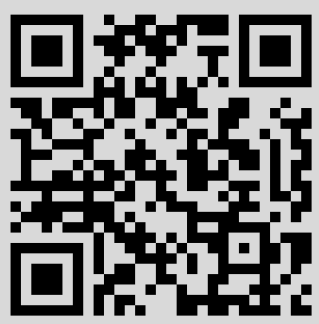




\title{
ВЫЧИСЛЕНИЕ ПЕРЕНОРМИРОВАННОЙ ДВУХТОЧЕЧНОЙ КОРРЕЛЯЦИОННОЙ ФУНКЦИИ МЕТОДОМ АДИАБАТИЧЕСКОЙ РЕГУЛЯРИЗАЦИИ
}

\begin{abstract}
В рамках метода адиабатической регуляризации вычислена перенормированная двухточечная корреляционная функция. Произведено детальное исследование случаев конформного и минимального взаимодействий для массивного и безмассового скалярных полей. Полученные ранее результаты выведены заново в строгой математической форме, дано объяснение некоторых эмпирических приближений и ограничений. Рассматриваются некоторые приложения к инфляционным моделям.
\end{abstract}

Ключевые слова: двухточечные функции, адиабатическая регуляризация, фаза де Ситтера.

\section{1. ВВЕДЕНИЕ}

Вычисление двухточечной корреляционной функции весьма важно с точки зрения инфляционных моделей. Например, в некоторых таких моделях (в модели новой инфляционной вселенной [1]-[3] и в сценарии хаотической инфляции [4]) возникает явление бесконечной инфляции, при котором квантовые флуктуации макроскопических масштабов для инфляционного поля, описываемые двухточечной корреляционной функцией, приводят к бесконечному процессу появления самовоспроизводящихся инфляционных мини-вселенных [5]. Другой пример связан с происхождением первоначальных возмущений и с их эволюцией [6], [7], исследование которой требует знания двухточечной корреляционной функции в импульсном пространстве (см., например, работу [8]). Наконец, исследования обратной реакции частиц, образовавшихся в фазе, предшествующей разогреву, также требуют знания двухточечных корреляционных функций инфляционного поля и светоподобных частиц, участвующих в этом процессе [9]-[11].

При вычислении двухточечных корреляционных функций главную трудность составляют ультрафиолетовые расходимости, которые требуется регуляризовать. Простейшим методом, позволяющим получать выражения, не содержащие расходимостей, оказывается адиабатическая регуляризация, основанная на вычитании неко-

${ }^{*}$ Departament de Matemàtica Aplicada I, Universitat Politècnica de Catalunya, Barcelona, Spain. E-mail: jaime.haro@upc.edu 
торых обобщенных мод Вентцеля-Крамера-Бриллюэна (ВКБ) [12], поведение которых в области высоких частот совпадает с поведением точных мод. Это позволяет сокращать расходящиеся члены. Однако в ранних работах на эту тему процедура вычисления перенормированных двухточечных корреляционных функций была несколько иной. Для получения конечных ответов авторам этих работ приходилось предполагать существование некоторого, не вполне обоснованного обрезания или производить необоснованные приближения [1], [13]-[15].

В современных работах применяется метод адиабатической регуляризации, хотя сама процедура остается не вполне ясной. При этом используется инфракрасное обрезание, остающееся и в окончательном выражении. Более того, в некоторых работах производятся вычитания адиабатических членов до четвертого порядка включительно, а вычисления интегралов производятся в рамках размерной регуляризации, что приводит к исключительно сложным выражениям [16]. Некоторые авторы для вычитания ультрафиолетовых расходимостей применяют сложный метод улучшенной адиабатической регуляризации [17].

Задача настоящей работы состоит в прояснении процедур, возникших в указанных работах, с математической точки зрения. Для этого исследуется скалярное поле, взаимодействующее с гравитацией минимальным и конформным образом. Случай безмассового поля исследуется в разделе 2 (случай конформного взаимодействия) и в разделе 3 (случай минимального взаимодействия), где все ранее полученные результаты выводятся подробно и очень простым образом. Например, показано, что при получении правильных ответов в случае минимального взаимодействия следует вычитать не все адиабатические моды, а лишь те из них, которые находятся внутри горизонта Хаббла. В разделе 4 исследуется массивное поле в фазе де Ситтера в предположении, что масса поля меньше, чем параметр Хаббла (эта ситуация типична для инфляционных моделей [5]). Дается подробный вывод двухточечной корреляционной функции, и производится строгое вычисление фундаментальной формулы (7), предложенной без вывода в работе [1]. Наконец, показано, что методология настоящей работы может быть применена и к более реалистичным моделям инфляции, в которых параметр Хаббла не постоянен, а представляет собой медленно меняющуюся величину.

\section{2. БЕЗМАССОВОЕ КОНФОРМНО ВЗАИМОДЕЙСТВУЮЩЕЕ ПОЛЕ}

Рассмотрим безмассовое конформно взаимодействующее скалярное поле, т. е. положим $m=0$ и $\xi=1 / 6$ в пространстве-времени Фридмана-Робертсона-Уокера $(\Phi Р У)$ и вычислим перенормированную часть двухточечной корреляционной функции $\left\langle\Phi^{2}(\eta, \vec{x})\right\rangle$, где $\eta$ - конформное время, с помощью метода адиабатической регуляризации. На этом простейшем примере удается продемонстрировать детали описываемого метода.

Общая теория квантованных полей в геометриях ФРУ хорошо изучена (подробнее см. монографию [18]). Рассмотрим скалярное поле

$$
\Phi(\eta, \vec{x})=\int_{\mathbb{R}^{3}} d^{3} k\left[a_{\vec{k}} u_{\vec{k}}(\eta, \vec{x})+a_{\vec{k}}^{\dagger} u_{\vec{k}}^{*}(\eta, \vec{x})\right],
$$


где

$$
u_{\vec{k}}(\eta, \vec{x})=\frac{e^{i \vec{k} \vec{x}}}{(2 \pi)^{3 / 2} \sqrt{C(\eta)}} \chi_{k}(\eta)
$$

$C$ - квадрат масштабного множителя $a, k \equiv|\vec{k}|$, а поле $\chi_{k}(\eta)$ удовлетворяет уравнению Клейна-Гордона

$$
\chi_{k}^{\prime \prime}+\Omega_{k}^{2}(\eta) \chi_{k}=0
$$

и условию Вронского $\chi_{k} \chi_{k}^{\prime *}-\chi_{k}^{*} \chi_{k}^{\prime}=i$, где $\Omega_{k}^{2}(\eta) \equiv k^{2}+C\left(m^{2}+(\xi-1 / 6) R\right), R-$ скалярная кривизна.

В случае безмассового поля с конформным взаимодействием моды имеют вид $\chi_{k}(\eta)=e^{-i k \eta} / \sqrt{2 k}$, и при этом двухточечная корреляционная функция задается выражением

$$
\left\langle\Phi^{2}(\eta, \vec{x})\right\rangle \equiv \int_{|\vec{k}| \leqslant \Lambda} d^{3} k\left|u_{\vec{k}}(\eta, \vec{x})\right|^{2}=\frac{1}{4 \pi^{2} C(\eta)} \int_{0}^{\Lambda} k d k
$$

где $\Lambda$ - обрезание по частотам, которое в конце вычислений следует устремить к бесконечности.

При получении перенормированного выражения для двухточечной корреляционной функции будем использовать метод Баниа, описанный в работе [12]. Сначала рассмотрим адиабатические моды, полученные в рамках приближения ВКБ,

$$
\chi_{k}=\frac{1}{\sqrt{2 W_{k} C}} \exp \left(-i \int W_{k} d \eta\right),
$$

до второго порядка включительно. Чтобы вычислить эти адиабатические моды до второго адиабатического порядка, подставим (5) в формулу (3), что приводит к уравнению [19], [20]

$$
W_{k}^{2}=\Omega_{k}^{2}-\frac{1}{2} \frac{W_{k}^{\prime \prime}}{W_{k}}+\frac{3}{4} \frac{\left(W_{k}^{\prime}\right)^{2}}{W_{k}^{2}} .
$$

Чтобы решить это уравнение, достаточно оставить только те члены, которые содержат самое бо́льшее две производные от скалярного множителя, т. е. только члены, содержащие $a,\left(a^{\prime}\right)^{2}$ и $a^{\prime \prime}$.

В случае конформного взаимодействия величина $W_{k}$ дается выражением (подробнее см. [21])

$$
W_{k}=\omega_{k}-\frac{1}{4} \frac{\omega_{k}^{\prime \prime}}{\omega_{k}^{2}}+\frac{3}{8} \frac{\left(\omega_{k}^{\prime}\right)^{2}}{\omega_{k}^{3}}
$$

где $\omega_{k}^{2}=k^{2}+C(\eta) m^{2}$, и с помощью простого вычисления получаем

$$
W_{k}=\omega_{k}-\frac{1}{8} \frac{m^{2} C^{\prime \prime}}{\omega_{k}^{3}}+\frac{5}{32} \frac{m^{4}\left(C^{\prime}\right)^{2}}{\omega_{k}^{5}} .
$$

После того как вычислены адиабатические моды, для построения перенормированного выражения для $\left\langle\Phi^{2}(\eta, \vec{x})\right\rangle$ необходимо вычесть из выражения (4) адиабатические члены до второго порядка включительно (только члены, содержащие не 
более двух производных, действующих на скалярный множитель), возникающие в выражении [7], [17]

$$
\frac{1}{4 \pi^{2} C(\eta)} \int_{0}^{\Lambda} \frac{k^{2}}{W_{k}} d k
$$

а затем взять предел при $m \rightarrow 0$, что дает

$$
\begin{aligned}
\left\langle\Phi^{2}(\eta, \vec{x})\right\rangle_{\text {ren }}= & \lim _{m \rightarrow 0} \frac{1}{4 \pi^{2} C(\eta)}\left[\int_{0}^{\Lambda}\left(k-\frac{k^{2}}{\omega_{k}}\right) d k+\right. \\
& \left.+\frac{m^{2} C^{\prime \prime}}{8} \int_{0}^{\Lambda} \frac{k^{2}}{\omega_{k}^{5}} d k-\frac{5 m^{4}\left(C^{\prime}\right)^{2}}{32} \int_{0}^{\Lambda} \frac{k^{2}}{\omega_{k}^{7}} d k\right] .
\end{aligned}
$$

Нетрудно показать, что окончательный ответ имеет вид

$$
\left\langle\Phi^{2}(\eta, \vec{x})\right\rangle_{\text {ren }}=-\frac{1}{96 \pi^{2} C}\left[\frac{1}{2}\left(\frac{C^{\prime}}{C}\right)^{2}-\frac{C^{\prime \prime}}{C}\right]=\frac{1}{48 \pi^{2}} \frac{a^{\prime \prime}}{a^{3}}=\frac{1}{288 \pi^{2}} R .
$$

Этот результат в фазе де Ситтера совпадает с ответом работы [22]. Отметим также, что для вселенной, заполненной излучением, в которой $R=0$, в безмассовом случае получим $\left\langle\Phi^{2}(\eta, \vec{x})\right\rangle_{\text {ren }}=0$ независимо от величины константы связи.

\section{3. БЕЗМАССОВОЕ ПОЛЕ С МИНИМАЛЬНЫМ ВЗАИМОДЕЙСТВИЕМ}

Рассмотрим другой простой пример, а именно безмассовое поле с минимальным взаимодействием в плоской карте пространства-времени де Ситтера, где моды также можно вычислить точно. Они имеют вид

$$
\varphi_{k}(\eta) \equiv \frac{\chi_{k}(\eta)}{\sqrt{C(\eta)}}=H \eta\left(a_{k} \psi_{k}(\eta)+b_{k} \psi_{k}^{*}(\eta)\right)
$$

где

$$
\psi_{k}(\eta)=-\sqrt{\frac{1}{2 k}} e^{-i k \eta}\left(1+\frac{1}{i k \eta}\right),
$$

$H$ - параметр Хаббла, а $a_{k}$ и $b_{k}$ - некоторые постоянные.

В общем случае выражение

$$
\left\langle\Phi^{2}(\eta, \vec{x})\right\rangle=\frac{1}{2 \pi^{2}} \int_{0}^{\Lambda} k^{2}\left|\varphi_{\vec{k}}(\eta)\right|^{2} d k
$$

содержит как ультрафиолетовые, так и инфракрасные расходимости. Чтобы исключить последние (см. подробное изложение в работах [23], [14]), рассмотрим переход из фазы с доминирующим излучением в фазу де Ситтера, описываемую следующим масштабным множителем:

$$
a(\eta)= \begin{cases}2-\frac{\eta}{\eta_{0}}, & \eta<\eta_{0} \\ \frac{\eta_{0}}{\eta}, & \eta>\eta_{0}\end{cases}
$$


где $\eta_{0}=-1 / H$.

Моды $\varphi_{k} \equiv \chi_{k} / \sqrt{C}$ при $\eta<\eta_{0}$ задаются выражениями $e^{-i k \eta} / \sqrt{2 k C}$. Заметим, что эти моды отвечают обычному выбору вакуумного состояния для безмассового поля в фазе излучения, так как скалярная кривизна обращается в нуль в этой моде; при этом уравнения движения мод $\chi_{k}$ имеют вид $\chi_{k}^{\prime \prime}+k^{2} \chi_{k}=0$, а вакуумное состояние соответственно получается тем же самым образом, что и в случае пространства Минковского, т. е. из мод $e^{-i k \eta}$.

Сопоставив моды и их производные, взятые в точке $\eta=\eta_{0}$, получим

$$
a_{k}=1+\frac{H}{i k}-\frac{H^{2}}{2 k^{2}}, \quad b_{k}=-\frac{H^{2}}{2 k^{2}} e^{2 i k / H}=a_{k}+\frac{2 i k}{3 H}+O\left(\frac{k^{2}}{H^{2}}\right) .
$$

При таком выборе коэффициентов имеем при малых значениях индекса $k$ в фазе де Ситтера $\left(\eta>\eta_{0}\right)$

$$
\left|\varphi_{k}\right|^{2}=\frac{H^{2} \eta^{2}}{2 k}\left[\left(\frac{2}{3 H \eta}+2+\frac{H^{2} \eta^{2}}{6}\right)^{2}+O\left(\frac{k^{2}}{H^{2}}\right)\right],
$$

что явно демонстрирует отсутствие инфракрасных расходимостей.

Для того чтобы проанализировать ультрафиолетовые расходимости, вычислим при больших $k$ величину

$$
\left|\varphi_{k}\right|^{2}=\frac{\eta^{2} H^{2}}{2 k}\left[1+\frac{1}{k^{2} \eta^{2}}-\frac{H^{2}}{k^{2}} \cos \left(2 k\left(H^{-1}+\eta\right)\right)+O\left(\frac{H^{3}}{k^{3}}\right)\right] .
$$

Из этого выражения следует, что члены, приводящие к ультрафиолетовым расходимостям в выражении для $\langle\Phi(\eta, \vec{x})\rangle$, имеют вид

$$
\frac{\eta^{2} H^{2}}{4 \pi^{2}} \int^{\Lambda} k d k, \quad \frac{H^{2}}{4 \pi^{2}} \int^{\Lambda} \frac{1}{k} d k
$$

так как с помощью интегрирования по частям можно показать, что интеграл

$$
-\frac{\eta^{2} H^{4}}{4 \pi^{2}} \int^{\Lambda} \frac{\cos \left(2 k\left(H^{-1}+\eta\right)\right)}{k} d k
$$

на самом деле сходится.

Теперь, когда расходящиеся члены выделены, можно вычислить адиабатические члены до второго порядка включительно. При $\eta>\eta_{0}$ эти члены имеют вид

$$
W_{k}=\omega_{k}-\frac{1}{\eta^{2} \omega_{k}}-\frac{1}{8} \frac{m^{2} C^{\prime \prime}}{\omega_{k}^{3}}+\frac{5}{32} \frac{m^{4}\left(C^{\prime}\right)^{2}}{\omega_{k}^{5}} .
$$

Отсюда можно заключить, что расходящиеся части в выражении (8) в фазе де Ситтера имеют вид

$$
\frac{\eta^{2} H^{2}}{4 \pi^{2}} \int^{\Lambda} \frac{k^{2}}{\omega_{k}} d k, \quad \frac{H^{2}}{4 \pi^{2}} \int^{\Lambda} \frac{k^{2}}{\omega_{k}^{3}} d k .
$$

Вычитая в области высоких частот расходящуюся часть выражения (8) из (4), например при $k>H$, получим

$$
\lim _{m \rightarrow 0} \frac{\eta^{2} H^{2}}{4 \pi^{2}} \int_{H}^{\Lambda}\left(k-\frac{k^{2}}{\omega_{k}}\right) d k=0, \quad \lim _{m \rightarrow 0} \frac{H^{2}}{4 \pi^{2}} \int_{H}^{\Lambda}\left(\frac{1}{k}-\frac{k^{2}}{\omega_{k}^{3}}\right) d k=0,
$$


откуда видно, что ультрафиолетовые расходимости сокращаются.

При этом, однако, возникает трудность, вызванная тем, что вычитаемый адиабатический член

$$
\frac{H^{2}}{4 \pi^{2}} \int_{0}^{H} \frac{k^{2}}{\omega_{k}^{3}} d k
$$

сам содержит инфракрасную расходимость, так как этот член расходится при приближении массы к нулю. Важно отметить, что этот же член можно записать в виде

$$
-\frac{R}{8 \pi^{2}} \int_{0}^{H}\left(\xi-\frac{1}{6}\right) \frac{k^{2}}{\omega_{k}^{3}} d k
$$

и он тем самым не проявляется в фазе конформного взаимодействия.

Решение проблемы этой инфракрасной расходимости вытекает из следующего наблюдения. Адиабатическое приближение основано на модах вида (5), и из самого́ вида этих функций следует, что они имеют смысл в том случае, когда точные моды $\chi_{k}$ имеют осциллирующий характер, т. е. когда $\chi_{k}$ удовлетворяют уравнению (3) с $\Omega_{k}^{2}(\eta)>0$.

Это условие в нашем случае означает, что $k>\sqrt{2}\left|\eta^{-1}\right|=\sqrt{2} H e^{H t}$, и тем самым необходимо вычитать только адиабатические моды, лежащие внутри горизонта Хаббла в момент времени $t$. Поэтому никаких инфракрасных расходимостей не возникает.

Сделаем важное замечание. Наш способ устранения инфракрасной расходимости не оказывает влияния на закон сохранения перенормированного тензора напряженности, который в метрике ФРУ задается выражением $\left(\rho a^{3}\right)^{\prime}+P\left(a^{3}\right)^{\prime}=0$, где $\rho-$ плотность энергии, а $P$ - давление, так как адиабатическая регуляризация состоит в процедуре вычитания адиабатических членов вплоть до указанного порядка, и при этом закон сохранения выполняется в каждом порядке по отдельности и, что еще важнее, такое вычитание может производиться по отдельности для каждой моды [24], [25]. Отсюда следует, что, обозначив через $\rho_{\mathrm{ad}}\left(\phi_{k}\right)$ и $P_{\mathrm{ad}}\left(\phi_{k}\right)$ соответствующие адиабатические члены для плотности энергии и давления, выраженные через адиабатические моды, определяемые формулой (5), получим, что условие сохранения $\left(\rho_{\mathrm{ad}}\left(\phi_{k}\right) a^{3}\right)^{\prime}+P_{\mathrm{ad}}\left(\phi_{k}\right)\left(a^{3}\right)^{\prime}=0$ будет выполнено, и, поскольку вычитания производятся в каждой моде по отдельности, можно вычесть заданное число мод, не нарушив при этом закон сохранения.

Подводя итог, приведем перенормированное выражение:

$$
\left\langle\Phi^{2}(\eta, \vec{x})\right\rangle_{\text {ren }}=\lim _{m \rightarrow 0} \frac{1}{2 \pi^{2}}\left(\int_{0}^{\Lambda} k^{2}\left|\varphi_{k}(\eta)\right|^{2} d k-\frac{1}{2 C} \int_{\sqrt{2} /|\eta|}^{\Lambda} \frac{k^{2}}{W_{k}} d k\right) .
$$

Теперь поскольку ультрафиолетовые расходимости сокращаются (см. (19)) и имеет место формула

$$
\lim _{m \rightarrow 0} \int_{\sqrt{2} /|\eta|}^{\Lambda} \frac{m^{2} k^{2}}{\omega_{k}^{5}} d k=\lim _{m \rightarrow 0} \int_{\sqrt{2} /|\eta|}^{\Lambda} \frac{m^{4} k^{2}}{\omega_{k}^{7}} d k=0,
$$


то с учетом (15) получим окончательное выражение:

$$
\begin{aligned}
\left\langle\Phi^{2}(\eta, \vec{x})\right\rangle_{\text {ren }}= & \frac{1}{2 \pi^{2}} \int_{0}^{\sqrt{2} /|\eta|} k^{2}\left|\varphi_{k}(\eta)\right|^{2} d k+ \\
& +\frac{\eta^{2} H^{2}}{4 \pi^{2}} \int_{\sqrt{2} /|\eta|}^{\infty}\left(-\frac{H^{2}}{k^{2}} \cos \left(2 k\left(H^{-1}+\eta\right)\right)+O\left(\frac{H^{3}}{k^{3}}\right)\right) k d k
\end{aligned}
$$

Если требуется исследовать поведение системы при больших временах $(\eta \cong 0$, т. е. $H t \gg 1)$, можно использовать следующее приближение:

$$
\left\langle\Phi^{2}(\eta, \vec{x})\right\rangle_{\text {ren }} \cong \frac{1}{2 \pi^{2}} \int_{0}^{\sqrt{2} /|\eta|} k^{2}\left|\varphi_{k}(\eta)\right|^{2} d k \cong \frac{1}{9 \pi^{2}} \int_{0}^{H} k d k+\frac{H^{2}}{4 \pi^{2}} \int_{H}^{\sqrt{2} /|\eta|} \frac{1}{k} d k
$$

где в первом интеграле было использовано приближение $\left|\varphi_{k}(\eta)\right|^{2} \cong 2 /(9 k)($ см. (14)), а во втором - приближение $\left|\varphi_{k}(\eta)\right|^{2} \cong H^{2} /\left(2 k^{3}\right)$ (см. (15)).

Наконец, после интегрирования получим, что при больших временах двухточечная корреляционная функция будет иметь вид

$$
\left\langle\Phi^{2}(\eta, \vec{x})\right\rangle_{\text {ren }} \cong \frac{H^{2}}{18 \pi^{2}}+\frac{H^{2}}{4 \pi^{2}}\left(\frac{1}{2} \ln 2+H t\right) \cong \frac{H^{3}}{4 \pi^{2}} t
$$

который совпадает с результатом, полученным ранее в работах [1], [13], [14], но только при больших временах, что разительно отличается от интерпретации этого результата, предложенной в работах [1], [2], [9], авторы которых утверждают, что поведение типа (24) характерно также и при малых временах в самом начале существования фазы де Ситтера.

Отметим также, что формула (23) оправдывает предписание работ [2], [26], где предполагается, что только моды вне горизонта Хаббла в момент времени $t$ дают вклад в величину перенормированной двухточечной корреляционной функции.

В противоположном случае, т. е. при малых временах Хаббла (например, при $t=1 / H)$ нетрудно показать, что $\left\langle\Phi^{2}(\eta, \vec{x})\right\rangle_{\text {ren }} \sim O\left(H^{2}\right)$.

В заключение настоящего раздела заметим, что в работе [7] исследовалась двухточечная корреляционая функция в чистой фазе де Ситтера, т. е. в отсутствие какого-либо фазового перехода. В качестве решения для отдельной моды использовалась функция (11) с параметрами $a_{k}=1$ и $b_{k}=0$, которая отвечает выбору вакуумного состояния Баниа-Девиса [27]. Автор утверждал, что в фазе де Ситтера перенормированная двухточечная корреляционная функция в точности обращается в нуль для безмассового поля с минимальным взаимодействием. Однако этот вывод был сделан на основе некорректных вычислений, в которых предел $m \rightarrow 0$ осуществлялся до интегрирования, что, разумеется, не позволило получить правильный ответ. Взятие предела $m \rightarrow 0$ до выполнения интегрирования в формуле (9) приводит к ответу $\left\langle\Phi^{2}(\eta, \vec{x})\right\rangle_{\text {ren }}=0$, который не совпадает с правильным результатом (10). 


\section{4. СЛУЧАЙ МАССИВНОГО ПОЛЯ}

Исследуем сначала случай с минимальным взаимодействием с $m \ll H$ в фазе де Ситтера ${ }^{1)}$. В разделе 3 было показано, что перенормированная двухточечная корреляционная функция задается формулой (20) до вычисления предела $m \rightarrow 0$, поскольку адиабатические моды, которые необходимо сократить, удовлетворяют условию

$$
k>\frac{\sqrt{2}}{|\eta|} \sqrt{1-\frac{m^{2}}{2 H^{2}}} \cong \frac{\sqrt{2}}{|\eta|} .
$$

Хотя вычисление двухточечной корреляционной функции в случае массивного поля оказывается более сложной задачей по сравнению со случаем безмассового поля, при больших временах можно, тем не менее, весьма точно аппроксимировать поведение массивного поля. Для этого заметим, что метод адиабатической регуляризации гарантирует, что интеграл

$$
\frac{1}{2 \pi^{2}} \int_{\sqrt{2} /|\eta|}^{\Lambda} k^{2}\left(\left|\varphi_{k}(\eta)\right|^{2}-\frac{1}{2 W_{k} C}\right) d k
$$

сходится [24]. Для выполнения интегрирования выберем решения для мод, которые отвечают вакуумному состоянию Баниа-Девиса, т. е.

$$
\varphi_{k}(\eta)=\sqrt{\frac{\pi}{4}} H \eta^{3 / 2} H_{\nu}^{(2)}(k \eta), \quad \nu \equiv \sqrt{\frac{9}{4}-\frac{m^{2}}{H^{2}}} \cong \frac{3}{2}-\frac{m^{2}}{3 H^{2}},
$$

где $H_{\nu}^{(2)}$ - функция Ханкеля (см., например, монографию [18]). Тогда при больших временах, используя асимптотическое разложение функции Ханкеля при больших аргументах (см. формулы (9.2.8)-(9.2.10) в [28]), можно показать, что расходящиеся члены в выражении (25) взаимно уничтожаются, и поскольку простые вычисления доказывают, что сходящиеся члены имеют порядок $O\left(\mathrm{~m}^{2}\right)$, их вкладом в двухточечную корреляционную функцию можно пренебречь. Тем самым при больших временах имеем

$$
\left\langle\Phi^{2}(\eta, \vec{x})\right\rangle_{\text {ren }} \cong \frac{1}{2 \pi^{2}} \int_{0}^{H} k^{2}\left|\varphi_{k}(\eta)\right|^{2} d k+\frac{1}{2 \pi^{2}} \int_{H}^{\sqrt{2} /|\eta|} k^{2}\left|\varphi_{k}(\eta)\right|^{2} d k .
$$

Чтобы исключить инфракрасные расходимости, вычислим первый из интегралов в предположении, что фазовый переход от вселенной с доминирующим излучением к вселенной в фазе де Ситтера происходит в момент времени $\eta_{0}=-1 / H$. Выполняя предельный переход при малом аргументе для функции Ханкеля при больших временах, авторы работ [26], [29] получили

$$
\frac{1}{2 \pi^{2}} \int_{0}^{H} k^{2}\left|\varphi_{k}(\eta)\right|^{2} d k \cong \frac{H^{2}}{8 \pi^{2}}
$$

\footnotetext{
1)Этот случай соответствует ситуации, в которой инфляционное поле находится в фазе медленного скатывания, и в этом режиме флуктуации скалярного поля, описываемые двухточечной корреляционной функцией, оказываются весьма существенными для понимания как процесса самовоспроизводящихся вселенных в инфляционных космологиях (см., например, [1], [2]), так и природы первоначальных возмущений, ответственных за анизотропию наблюдаемой вселенной.
} 
Тем не менее мы не можем согласиться с этим результатом, так как он основан на формуле (см. формулу (А44) работы [26])

$$
\left|a_{k}-b_{k}\right|^{2}=\left(1+\left(\frac{2^{2 \nu}}{2 \pi}\right)\left(-\frac{k}{H}\right)^{1-2 \nu} \Gamma^{2}(\nu)\right)^{-1}
$$

в которой $a_{k}$ и $b_{k}$ суть коэффициенты, входящие в нижеследующую формулу (29), которая не обеспечивает хорошего приближения. Чтобы это доказать, применим эту формулу в безмассовом случае, т. е. при $\nu=3 / 2$. Тогда результат при малых значениях $k$ имеет вид $\left|a_{k}-b_{k}\right|^{2} \cong k^{2} / H^{2}$, который не совпадает с корректным ответом, получаемым из формулы (13) настоящей работы. На самом деле, применяя (13), получим, что при малых значениях индекса $k$ имеет место соотношение $\left|a_{k}-b_{k}\right|^{2} \cong 4 k^{2} / 9 H^{2}$.

Для получения правильного ответа необходимо рассмотреть общие решения для мод в фазе де Ситтера

$$
\varphi_{k}(\eta)=\sqrt{\frac{\pi}{4}} H \eta^{3 / 2}\left(a_{k} H_{\nu}^{(2)}(k \eta)+b_{k} H_{\nu}^{(1)}(k \eta)\right)
$$

и установить соответствие между модами и их производными по времени в точке $\eta_{0}=-1 / H$, что дает

$$
\begin{aligned}
a_{k} & =\frac{1}{2 i} \sqrt{\frac{\pi k \eta_{0}}{2}}\left(\left(-i+\frac{H}{2 k}\right) H_{\nu}^{(1)}\left(k \eta_{0}\right)-H_{\nu}^{(1)^{\prime}}\left(k \eta_{0}\right)\right) e^{i k / H}, \\
b_{k} & =-\frac{1}{2 i} \sqrt{\frac{\pi k \eta_{0}}{2}}\left(\left(-i+\frac{H}{2 k}\right) H_{\nu}^{(2)}\left(k \eta_{0}\right)-H_{\nu}^{(2)^{\prime}}\left(k \eta_{0}\right)\right) e^{i k / H} .
\end{aligned}
$$

Применяя теперь формулу (см. [28])

$$
H_{\nu}^{(1,2)^{\prime}}(z)=H_{\nu-1}^{(1,2)}(z)-\frac{\nu}{z} H_{\nu}^{(1,2)}(z)
$$

получим

$$
\left|a_{k}-b_{k}\right|=\sqrt{\frac{\pi k}{2 H}}\left|J_{\nu-1}\left(k \eta_{0}\right)-\left(-i+\frac{H}{2 k}-\frac{\nu H}{k}\right) J_{\nu}\left(k \eta_{0}\right)\right|
$$


где $J_{\nu}=\left(H_{\nu}^{(1)}+H_{\nu}^{(2)}\right) / 2-$ функция Бесселя первого рода. Тогда в пределе малых аргументов

$$
J_{\nu}\left(k \eta_{0}\right) \cong \frac{1}{\Gamma(\nu+1)}\left(\frac{k \eta_{0}}{2}\right)^{\nu}, \quad H_{\nu}^{(2)}\left(k \eta_{0}\right) \cong-H_{\nu}^{(1)}\left(k \eta_{0}\right) \cong \frac{i}{\pi} \Gamma(\nu)\left(\frac{k \eta_{0}}{2}\right)^{-\nu}
$$

получим, что

$$
\left|\varphi_{k}(\eta)\right|^{2} \cong \frac{2}{9 k}(H|\eta|)^{3-2 \nu}
$$

и, наконец, придем к окончательному ответу:

$$
\frac{1}{2 \pi^{2}} \int_{0}^{H} k^{2}\left|\varphi_{k}(\eta)\right|^{2} d k \cong \frac{H^{2}}{18 \pi^{2}} e^{-2 m^{2} t / 3 H} \cong \begin{cases}\frac{H^{2}}{18 \pi^{2}}, & \frac{m^{2} t}{H} \ll 1 \\ 0, & \frac{m^{2} t}{H} \gg 1\end{cases}
$$

который согласуется с первым из интегралов в правой части формулы (23).

Второй из этих интегралов можно вычислить, применив следующее приближение, справедливое при $k>H$ :

$$
\varphi_{k}(\eta) \cong \sqrt{\frac{\pi}{4}} H \eta^{3 / 2} H_{\nu}^{(2)}(k \eta)
$$

Из (30) легко получить, что эффективно можно считать $a_{k} \cong 1$ и $b_{k} \cong 0$ в области $k>H$. Тогда, поскольку при больших временах имеет место соотношение

$$
H_{\nu}^{(2)}(k \eta) \cong-\frac{i}{\pi} \Gamma(\nu)\left(\frac{k \eta}{2}\right)^{-\nu}
$$

подставляя это выражение во второй из интегралов формулы (27), получим для массивного поля с минимальным взаимодействием следующее выражение в предположении $m \ll H$ :

$$
\begin{aligned}
\frac{1}{2 \pi^{2}} \int_{H}^{\sqrt{2} /|\eta|} k^{2}\left|\varphi_{k}(\eta)\right|^{2} d k & \cong \frac{3 H^{4}}{8 m^{2} \pi^{2}}\left[2^{m^{2} / 3 H^{2}}-(H|\eta|)^{2 m^{2} / 3 H^{2}}\right] \cong \\
& \cong \frac{3 H^{4}}{8 m^{2} \pi^{2}}\left[1-e^{-2 m^{2} t / 3 H}\right] .
\end{aligned}
$$

Поскольку первый из интегралов в правой части формулы (27) меньше второго интеграла, в зависимости от величины $m^{2} t / H$ получим следующие аппроксимирующие выражения:

$$
\left\langle\Phi^{2}(\eta, \vec{x})\right\rangle_{\text {ren }} \cong \frac{3 H^{4}}{8 m^{2} \pi^{2}}\left[1-e^{-2 m^{2} t / 3 H}\right] \cong \begin{cases}\frac{H^{3}}{4 \pi^{2}} t, & \frac{m^{2} t}{H} \ll 1 \\ \frac{3 H^{4}}{8 m^{2} \pi^{2}}, & \frac{m^{2} t}{H} \gg 1\end{cases}
$$

которые совпадают при больших временах с формулой (7) работы [1]. Заметим, что автор работы [1] привел формулу без вывода, отметив лишь то, что вычисления были весьма трудоемкими. 
Вычислим, наконец, перенормированную двухточечную корреляционную функцию для массивного конформно взаимодействующего поля в режиме $m \ll H$ в фазе де Ситтера, задаваемую выражением²)

$$
\left\langle\Phi^{2}(\eta, \vec{x})\right\rangle_{\mathrm{ren}}=\frac{1}{2 \pi^{2}}\left(\int_{0}^{\Lambda} k^{2}\left|\varphi_{k}(\eta)\right|^{2} d k-\frac{1}{2 C} \int_{0}^{\Lambda} \frac{k^{2}}{W_{k}} d k\right),
$$

в котором величина $W_{k}$ задается формулой (6).

При больших временах можно применить те же рассуждения, которые были использованы в случае, когда мы пренебрегли некоторыми членами, возникающими в (25). При этом получается следующее приближение:

$$
\left\langle\Phi^{2}(\eta, \vec{x})\right\rangle_{\text {ren }} \cong \frac{1}{2 \pi^{2}}\left(\int_{0}^{A|\eta|^{-1}}\left|\varphi_{k}(\eta)\right|^{2} d k-\frac{1}{2 C} \int_{0}^{A|\eta|^{-1}} \frac{k^{2}}{W_{k}} d k\right),
$$

в котором $A$ - некоторая безразмерная постоянная порядка единицы. На практике можно положить $A=1$; рассуждения при этом не изменятся, так как величина $|\eta|^{-1}$ оказывается достаточно большой при больших временах.

Поскольку никаких инфракрасных расходимостей в этом случае не появляется, можно применить разложение по модам (26), отвечающим вакуумному состоянию Баниа-Девиса. Тогда при $k<A /|\eta|$ хорошим приближением оказывается $\varphi_{k}(\eta) \cong$ $-i H \eta / \sqrt{2 k}$, и поскольку

$$
\frac{H^{2} \eta^{2}}{4 \pi^{2}} \int_{0}^{A|\eta|^{-1}}\left(k-\frac{k^{2}}{\omega_{k}}\right) d k \sim O\left(m^{2} \ln \left(\frac{H}{m}\right)\right),
$$

то этим членом можно пренебречь (он оказывается малым по сравнению с другими членами, которые имеют порядок $\left.O\left(H^{2}\right)\right)$; при больших временах получается тот же самый результат, что и в формуле (10) при $R=12 H^{2}$, т. е.

$$
\left\langle\Phi^{2}(\eta, \vec{x})\right\rangle_{\mathrm{ren}}=\frac{H^{2}}{24 \pi^{2}}
$$

так как ведущие члены в выражении (38) совпадают с ведущими членами в формуле $(9)$.

Сделаем заключительное замечание. Если рассмотреть вакуумные флуктуации инфляционного поля в фазе де Ситтера с минимальным взаимодействием и в режиме $m \ll H$, то его двухточечную корреляционную функцию, а именно $\left\langle\Phi_{\inf }^{2}(\eta, \vec{x})\right\rangle_{\text {ren }}$, можно аппроксимировать выражением (36). С другой стороны, если рассмотреть процесс создания светоподобных частиц гравитационными частицами посредством почти конформного взаимодействия [30]-[32], то двухточечная корреляционная функция $\left\langle\Phi_{\text {part }}^{2}(\eta, \vec{x})\right\rangle_{\text {ren }}$ будет приближенно задаваться формулой (40). Тем самым при больших временах имеем $\left\langle\Phi_{\text {inf }}^{2}(\eta, \vec{x})\right\rangle_{\text {ren }} \gg\left\langle\Phi_{\text {part }}^{2}(\eta, \vec{x})\right\rangle_{\text {ren }}$, что означает, что в фазе де Ситтера флуктуации поля частиц оказываются меньше, чем флуктуации инфляционного поля, а следовательно, первые флуктуации не оказывают влияния на

2) Заметим, что в случае конформного взаимодействия не требуется пренебрегать никакими адиабатическими модами. 
процесс инфляции. Отсюда можно заключить, что поскольку на протяжении периода медленного скатывания параметр Хаббла меняется медленно, то этот результат, по-видимому, будет справедлив и для более физически значимых инфляционных моделей.

Чтобы это доказать, рассмотрим модель хаотической инфляции с потенциалом $V=M^{2} \phi^{2} / 2$ [4]. В режиме медленного скатывания имеем

$$
\phi(t) \cong \phi_{0}-\frac{M M_{\mathrm{Pl}}}{2 \sqrt{3 \pi}} t, \quad H(t) \cong H_{0}-\frac{M^{2}}{3} t,
$$

где $M_{\mathrm{Pl}}$ - масса Планка [5]. Предполагая, что инфляция начинается в планковскую эру, т. е. $H_{0} \sim M_{\mathrm{Pl}}$, и применяя уравнение Фридмана

$$
H_{0}^{2} \cong \frac{8 \pi V\left(\phi_{0}\right)}{3 M_{\mathrm{Pl}}^{2}}
$$

получим, что $\phi_{0} \sim M_{\mathrm{Pl}}^{2} / M$. Поскольку режим медленного скатывания заканчивается при $\phi(t) \sim M_{\mathrm{Pl}}$, получим, что инфляция завершается при временах $t_{\mathrm{f}} \sim H_{0} / M^{2}$. Если в такой модели инфляция завершается на энергиях порядка энергии Великого Объединения, то получим, что $M \sim 10^{-5} M_{\mathrm{Pl}}$, и тем самым можно заключить, что $M \ll H_{0}$. Более того, при $0<t \ll H_{0} / M^{2}$ режим медленного скатывания совпадает с фазой де Ситтера, и потому наши результаты применимы при описании этого режима. На самом деле решения для мод массивного минимально взаимодействующего поля массы $m$ в эпоху, когда $0<t \ll H_{0} / M^{2}$, с хорошей точностью задаются формулами

$$
\varphi_{k}(\eta)=\sqrt{\frac{\pi}{4}} H_{0} \eta^{3 / 2}\left(a_{k} H_{\nu}^{(2)}(k \eta)+b_{k} H_{\nu}^{(1)}(k \eta)\right),
$$

где $\nu=\sqrt{9 / 4-\left(m^{2}-M^{2}\right) / H_{0}^{2}}$ (см. формулы (6) и (14) работы [16]). Применяя аргументацию, использованную в начале этого раздела, получим, что если обе величины $M$ и $m$ меньше, чем $H_{0}$, то в эпоху $1 / H_{0} \ll t \ll H_{0} / M^{2}$ формула (40) остается справедливой и для почти конформно взаимодействующего поля. С другой стороны, ведущий член двухточечной корреляционной функции для инфляционного поля имеет вид $\left\langle\Phi^{2}(\eta, \vec{x})\right\rangle \cong H_{0}^{3} t / 4 \pi^{2}$, что доказывает наше предыдущее утверждение.

\section{5. ЗАКЛЮЧЕНИЕ}

В работе детально исследован и прояснен с математически строгой точки зрения адиабатический метод перенормировки двухточечной корреляционной функции. Предшествующие результаты, полученные в рамках этого метода, выведены заново и обоснованы, что необходимо, особенно с учетом того обстоятельства, что в литературе на эту тему встречается довольно много неясных приближений, которые требовали прояснения. Полученные результаты могут быть применены к реалистичным инфляционным моделям при исследовании задачи обратного влияния процесса рождения частиц в конце периода инфляции, поскольку решение задач указанного типа основано на вычислении двухточечных корреляционных функций (см., например, [11], [16]). 
Благодарности. Работа выполнена при частичной финансовой поддержке MICINN (Испания), проект MTM2008-06349-C03-01 и AGAUR (провинция Каталония), контракт 2005SGR-00790. Исследование было частично выполнено в Институте космических исследований Каталонии (Автономный университет г. Барселоны, Испания).

\section{Список литературы}

[1] A. D. Linde, Phys. Lett. B, 116:5 (1982), 335-339.

[2] A. Vilenkin, Phys. Rev. D, 27:12 (1983), 2848-2855.

[3] S. W. Hawking, I. G. Moss, Nucl. Phys. B, 224:1 (1983), 180-192.

[4] A. D. Linde, Phys. Lett. B, 129:3-4 (1983), 177-181.

[5] A. D. Linde, Phys. Lett. B, 162:4-6 (1985), 281-286.

[6] A.R. Liddle, D.H. Lyth, Cosmological Inflation and Large-Scale Structure, Cambridge Univ. Press, Cambridge, 2000.

[7] L. Parker, Amplitude of perturbations from inflation, arXiv: hep-th/0702216v2.

[8] R. H. Brandenberger, Rev. Mod. Phys., 57 (1985), 1.

[9] G. Felder, L. Kofman, A. Linde, Phys. Rev. D, 60:10 (1999), 103505, 10 pp., arXiv: hep-ph/9903350.

[10] P. R. Anderson, C. Molina-París, D. Evanich, G. B. Cook, Phys. Rev. D, 78:8 (2008), 083514, 18 pp., arXiv: 0801.0730.

[11] F. Finelli, G. Marozzi, A. A. Starobinsky, G. P. Vacca, G. Venturi, Phys. Rev. D, 79:4 (2009), 044007, 9 pp., arXiv: 0808.1786.

[12] T.S. Bunch, J. Phys. A, 11:3 (1978), 603-607.

[13] A. A. Starobinsky, Phys. Lett. B, 117:3-4 (1982), 175-178.

[14] A. Vilenkin, L. H. Ford, Phys. Rev. D, 26:6 (1982), 1231-1241.

[15] A. Vilenkin, Nucl. Phys. B, 226:2 (1983), 527-546.

[16] F. Finelli, G. Marozzi, G. P. Vacca, G. Venturi, Phys. Rev. D, 65:10 (2002), 103521, 14 pp.

[17] C. Molina-París, P. R. Anderson, S. A. Ramsey, Phys. Rev. D, 61:12 (2000), 127501, 4 pp., arXiv: gr-qc/9908037.

[18] N. D. Birrell, C. P. W. Davies, Quantum Fields in Curved Space, Cambridge Monogr. Math. Phys., 7, Cambridge Univ. Press, Cambridge, 1982.

[19] P. R. Anderson, L. Parker, Phys. Rev. D, 36:10 (1987), 2963-2969.

[20] S. Habib, C. Molina-París, E. Mottola, Phys. Rev. D, 61:2 (1999), 024010, 22 pp., arXiv: gr-qc/9906120.

[21] S. Winitzki, Phys. Rev. D, 72:10 (2005), 104011, 14 pp., arXiv: gr-qc/0510001.

[22] B. Allen, A. Folacci, Phys. Rev. D, 35:12 (1987), 3771-3778.

[23] L. H. Ford, L. Parker, Phys. Rev. D, 16:2 (1977), 245-250.

[24] L. Parker, S. A. Fulling, Phys. Rev. D, 9:2 (1974), 341-354.

[25] T.S. Bunch, J. Phys. A, 13:4 (1980), 1297-1310.

[26] S. Habib, Phys. Rev. D, $46: 6$ (1992), 2408-2427, arXiv: gr-qc/9208006.

[27] T. S. Bunch, P. C. W. Davies, Proc. Roy. Soc. A, 360:1700 (1978), 117-134.

[28] М. Абрамовиц, И. Стегун (ред.), Справочник по специалъным функииям с формулами, графиками и математическими таблицами, Наука, М., 1979.

[29] V. Sahni, Class. Quantum Grav., 5:7 (1988), L113-L118.

[30] A. Vilenkin, Phys. Rev. D, 32:10 (1985), 2511-2521.

[31] L. H. Ford, Phys. Rev. D, 35:10 (1987), 2955-2960.

[32] S. Spokoiny, Phys. Lett. B, 315:1-2 (1993), 40-45, arXiv: gr-qc/9306008.

Поступила в редакцию 11.12.2009, после доработки 11.02.2010 